\title{
An Evaluation of Radiographic Hepatic Size in Dogs with Portosystemic Shunt
}

\author{
Makoto WASHIZU $^{1)}$, Michio KATAGI ${ }^{1)}$, Tsukimi WASHIZU ${ }^{2}$, Shido TORISU ${ }^{1)}$, Yogo KONDO $^{1)}$ and Ayami NOJIRI ${ }^{1)}$ \\ ${ }^{1)}$ Laboratory of Veterinary Medical Teaching Hospital and ${ }^{2)}$ Department of Clinical Pathology, Nippon Veterinary and Animal Science \\ University, 1-7-1 kyonan-cho Musashino-shi, Tokyo 180-8602 Japan
}

(Received 11 June 2003/Accepted 9 March 2004)

ABSTRACT. Radiographically, the hepatic sizes of portosystemic shunt (PSS) cases were evaluated. In this study the hepatic area was compared in PSS and non-PSS dogs by utilizing the right lateral radiography. The top three breeds of PSS dogs of Maltese, Shih Tzu and Yorkshire Terrier, were included and these dogs had a significantly smaller hepatic area ratio of $46.37 \pm 0.63 \%, 61.76 \pm 0.78 \%$ and 41.59 $\pm 0.23 \%$ respectively $(\mathrm{p}<0.05)$ and the average overall hepatic area in the 3 dog breeds was $47.75 \pm 0.40 \%$.

KEY WORDS: canine, hepatic size, portosystemic shunt.

J. Vet. Med. Sci. 66(8): 977-978, 2004

Microhepatica is a typical finding when evaluating abdominal radiographs of patients with portosystemic shunt (PSS) [1, 2]. To date, however, no research has been conducted to compare and actually quantify the hepatic size differences between PSS and non-PSS dogs. Diseased states in which microhepatica may be diagnosed through radiographs include cirrhosis, hepatic fibrosis, congenital portosystemic shunt and diaphragmatic hernia in which the liver is partially or completely contained within the chest [4]. The radiographic diagnosis of PSS is usually based on the small liver or microhepatica. The present study evaluates the area of the liver on the X-ray films in dogs diagnosed as having congenital PSS. The evaluation of the hepatic area was performed with NIH Image software [3] to process and edit radiographic images. Hepatic areas in non-PSS dogs and dogs with PSS were photographed with a digital camera utilizing radiograph films. Hepatic areas were ascertained by counting numbers of digital dots and obtained a proportional value for the hepatic area.

A comparative study was made with three breeds of dogs: Maltese, Shih Tzu, and Yorkshire Terriers. These breeds were selected since they had a higher rate of occurrence in 150 PSS surgical cases that were operated at the Nippon Veterinary and Animal Science University from 1990 to 2003. This study was aimed to quantify the actual hepatic area of PSS affected dogs compared with non-PSS canine hepatic size. The diagnosis of PSS were made on the basis of typical clinical signs of hepatic encephalophathy, biochemical profiles of increased ammonia levels and serum bile acids, decreased BUN and Albumin, radiographic and echographic evidences of small liver mass and poor development of portal veins in hepatic parenchyma. Finally, the presence of PSS was confirmed by the portogram and the shunts were ligated either completely or partially occluded by means of Ameriod Constrictor or cellophane bandage.

Plain right lateral radiographs of non-PSS Maltese $(n=9$, median age $=15$ months, body weight $=3.37 \pm 0.87 \mathrm{~kg}$ ), nonPSS Shih Tzu $(n=8$, median age $=13$ months, body weight $=4.30 \pm 1.37 \mathrm{~kg}$ ), and non-PSS Yorkshire Terriers $(\mathrm{n}=11$, median age $=12$ months, body weight $=2.15 \pm 0.53$ $\mathrm{kg}$ ) were employed in the analysis of the hepatic area in dogs which had radiographs of the upper abdomen as controls. These control dogs as shown as non-PSS dogs did not have any clinical signs of illness and did not have any hematological or biochemical abnormalities. Plain right lateral radiographs of Maltese $(n=12$, median age $=10$ months, body weight $=2.44 \pm 1.05 \mathrm{~kg})$, Shih Tzu $(\mathrm{n}=8$, median age $=18$ months, body weight $=4.33 \pm 1.27 \mathrm{~kg}$ ), and Yorkshire Terriers $(n=10$, median age $=20$ months, body weight $=2.00 \pm 0.90$ $\mathrm{kg}$ ) were employed in the analysis of the hepatic area in dogs with PSS.

The following method was employed to measure the radiographic hepatic area. One right lateral radiograph of each of the dogs was employed in the study. Each film was taken by a digital camera with an affixed metric measuring scale. In order to correct for differences in the hepatic area due to variation in body weight between individuals, an image of the patient's fourth lumbar vertebra, which is easily measured, was traced onto the computer screen and the area of the vertebra was measured. The traced portion was then copied and pasted onto NIH Image software, and the number of pixels in the image was calculated. The number of pixels on the measuring scale was converted to centimeter dimensions, and the area of the measured fourth lumbar vertebra was then calculated in centimeters with this conversion scale. The silhouette of the liver was then traced and its area calculated in the same way. The radiographic hepatic area ratio was calculated with the formula: Hepatic area ratio= liver area/fourth lumbar vertebra area.

Each individual's hepatic area ratio was obtained. A proportional value $(\%)$ for the hepatic area in PSS dogs with respect to normal dogs was then obtained with the formula: (PSS dog/ hepatic area ratio)/(normal dog/ hepatic area ratio) $\times 100$.

Student's $t$-test was performed for normal and PSS dogs and the hepatic area ratio of PSS dogs in all three breeds was confirmed to be clearly smaller than that of normal dogs.

The fourth lumber vertebra area, hepatic area, hepatic area ratio and the hepatic area proportion in normal and PSS dogs are shown on Table 1. The proportional hepatic areas 
Table 1. Measurements results for hepatic area, fourth lumber vertebrae areas and Proportion in normal and PSS dogs in Maltese, Shih Tzu and Yorkshire Terrier (mean \pm SE)

\begin{tabular}{|c|c|c|c|c|}
\hline Breed & $\begin{array}{l}\text { Fourth lumbar } \\
\text { vertebrae area } *\end{array}$ & $\begin{array}{l}\text { Hepatic area } \\
*\end{array}$ & $\begin{array}{l}\text { Hepatic ratio } \\
\text { (liver/lumbar } \\
\text { vertebrae)* }\end{array}$ & $\begin{array}{c}\text { Hepatic area } \\
\text { proportion } \\
(\%)\end{array}$ \\
\hline $\begin{array}{l}\text { Maltese normal } \\
(\mathrm{n}=9)\end{array}$ & $1.15 \pm 0.13$ & $22.23 \pm 3.54$ & $19.33 \pm 2.89$ & \\
\hline $\begin{array}{l}\text { Maltese PSS } \\
(\mathrm{n}=12)\end{array}$ & $1.10 \pm 0.23$ & $9.75 \pm 3.01 * *$ & $8.86 \pm 2.52^{* *}$ & $46.37 \pm 0.63 * *$ \\
\hline $\begin{array}{c}\text { Shih Tzu } \\
\text { normal }(\mathrm{n}=8)\end{array}$ & $1.27 \pm 0.23$ & $21.12 \pm 4.31$ & $16.90 \pm 3.55$ & \\
\hline $\begin{array}{l}\text { Shih Tzu PSS } \\
(\mathrm{n}=8)\end{array}$ & $1.29 \pm 0.27$ & $12.32 \pm 1.36^{* *}$ & $10.53 \pm 2.92 * *$ & $61.76 \pm 0.78^{* *}, * * *$ \\
\hline $\begin{array}{l}\text { Yorkshire Terrier } \\
\text { Normal }(\mathrm{n}=11)\end{array}$ & $0.85 \pm 0.20$ & $19.19 \pm 4.57$ & $22.57 \pm 6.73$ & \\
\hline $\begin{array}{l}\text { Yorkshire Terrier } \\
\text { PSS }(n=10)\end{array}$ & $0.86 \pm 0.12$ & $8.02 \pm 1.91^{* *}$ & $9.96 \pm 1.75^{* *}$ & $41.59 \pm 0.23^{* *}$ \\
\hline $\begin{array}{l}\text { Three breeds } \\
\text { average }\end{array}$ & & & & $47.75 \pm 0.04$ \\
\hline
\end{tabular}

of Maltese, Shith Tzu and Yorkshire Terrier were $46.37 \pm$ $0.63 \%, 61.76 \pm 0.78 \%$ and $41.59 \pm 0.23 \%$ respectively and these 3 canine breed's hepatic size proportions were significantly smaller than the those of normal dogs $(\mathrm{p}<0.05)$. The average of the three breed of dogs was $47.75 \pm 0.40 \%$. There was a significant difference in the hepatic area proportion between Shih Tzu and Maltese $(\mathrm{p}<0.05)$ and between Shih Tzu and Yorkshire Terrier $(\mathrm{p}<0.05)$.

The radiographs employed in the measurement of the hepatic area for the current study were selected from among 150 PSS canine cases. These were based on the clarity of the hepatic silhouette and the ease with which this silhouette could be traced onto a computer screen. A variety of methods have been reported for the measurement of liver size from plain radiographs. R. Lee et al. [5] employed a formula to evaluate liver length and thickness. Heni van Bree et al. [6] calculated liver volume from a formula that is not affected by the inspiratory/expiratory phase of the thorax. These methods, however, are for measuring hepatic size by using the silhouette of a normal liver. The rounding of the edges in a PSS liver can make it difficult to determine length from the hepatic edge to the peak, and accordingly the aforementioned formulas could not be applied. Therefore, in this study, NIH Image software was used to determine the hepatic size in normal and PSS dogs. With this software, an outline of the liver could be traced onto radiographic film, so that the hepatic area ratio could be determined regardless of whether the hepatic edges where rounded or not. The hepatic area proportion for PSS dogs could be expressed as a number. With normal dogs designated as being $100 \%$, the hepatic area ratio in PSS dogs was found to be smaller. Namely, the hepatic area proportion in Maltese, Shih Tzu, and Yorkshire Terriers was 46\%, 63\% and 44\%, respectively and the average liver area in PSS dogs was almost $47 \%$ of the normal hepatic size in the right lateral radiographic view. The disparity in the values for the Shih Tzu versus the other two breeds can be explained by the fact that a normal Shih Tzu had a smaller hepatic area ratio than that of normal Maltese and Yorkshire Terriers, and the PSS Shih Tzu had a larger hepatic area ratio than that of the PSS Maltese and Yorkshire Terriers. For this reason, the hepatic area ratio was also larger and the hepatic size proportion was greater than that of the other two dog breeds. This is because the area of the fourth lumbar vertebra is large in both normal and PSS Shih Tzu, and because the hepatic area is also large in the PSS Shih Tzu.

\section{REFERENCES}

1. Lee R. Manual of Small Animal Diagnostic Imaging Second Edition. Chapter4:85. BSAVA,Gloucestershire.

2. Lee R., C. Leowijuk. 1982. J. Small Anim. Pract. 23: 251-269.

3. NIH image: http://www.scioncorp.com/

4. Richard W. Nelson, Guillermo Couto C. Small Animal Internal Medicine Second Edition. Chapter 36: 497-499 Mosby. St. Louis, Missouri.

5. Robert D. Pechman, Jr. The Liver and Spleen.: Text Book of veterinary Diagnostic Radiology, Chapter 38: 458-461.

6. Henri van Bree, DVM. Veerle Jacobs, DVM et al, 1989. Am. J. Vet. Res. 50: 1613-1615. 